\title{
Rabdomiossarcoma botrióide na vesícula urinária de um American Pitbull Terrier
}

\author{
Rhabdomyosarcoma in the urinary bladder of an American Pitbull Terrier \\ Rabdomiosarcoma botrioide en la vejiga urinaria de un American Pittbull Terrier
}

Recebido: 09/04/2021 | Revisado: 20/04/2021 | Aceito: 27/04/2021 | Publicado: 12/05/2021

\author{
Melissa Cristina Müller \\ ORCID: https://orcid.org/0000-0003-0785-7464 \\ Universidade Federal do Vale do São Francisco, Brasil \\ E-mail: mullercm9@gmail.com \\ Ana Catarina Luscher Albinati \\ ORCID: https://orcid.org/0000-0003-2954-3431 \\ Universidade Federal do Vale do São Francisco, Brasil \\ E-mail: catarina.albinati@gmail.com \\ Durval Baraúna Júnior \\ ORCID: https://orcid.org/0000-0002-0261-6672 \\ Universidade Federal do Vale do São Francisco, Brasil \\ E-mail: durvalbarauna@ hotmail.com \\ Cássia Regina Oliveira Santos \\ ORCID: https://orcid.org/0000-0002-8676-6095 \\ Universidade Federal do Vale do São Francisco, Brasil \\ E-mail: cassiareginavet@yahoo.com.br \\ Eliane Feitosa Ferreira \\ ORCID: https://orcid.org/0000-0002-6691-8127 \\ Universidade Federal do Vale do São Francisco, Brasil \\ E-mail: elianevet@outlook.com \\ Wasley Carlos Gonçalves de Matos \\ ORCID: https://orcid.org/0000-0002-3799-1122 \\ Universidade Federal do Vale do São Francisco, Brasil \\ E-mail: wasley.matos@univasf.edu.br
}

\begin{abstract}
Resumo
O rabdomiossarcoma botrióide é uma variante do rabdomiossarcoma embrionário e tem origem nas células musculares estriadas, ou precursores destas células, por isso podem se iniciar em partes do corpo com ausência de músculo estriado, como o trato urogenital. Geralmente, os cães acometidos são jovens até dois anos e as massas neoplásicas têm aspecto de cacho de uva. O objetivo do presente relato foi descrever um caso de rabdomiossarcoma botrióide em um cão, American Pittbull Terrier, de 16 meses que apresentou uma massa no interior da vesícula urinária, posteriormente diagnosticada através de exame histopatológico e imunoistoquímico. A massa era multilobulada, firme, rósea a vermelha com áreas multifocais de necrose e estava aderida na mucosa da região mais cranial da vesícula urinária emitindo ramificações disseminadas em direção ao trígono vesical. Microscopicamente, a neoplasia é constituída por duas populações celulares entremeadas a uma matriz mixóide, uma composta por células pequenas e redondas, com citoplasma escasso e núcleo basofílico hipercorado e a outra por células alongadas, com citoplasma eosinofílico e núcleo paracentral, ambas as populações celulares possuíam pleomorfismo moderado e 2 figuras de mitose por campo (400 x). Densidade celular aumentada foi observada abaixo do epitélio vesical. A combinação de exames complementares foi conclusiva para o diagnóstico da neoplasia.
\end{abstract}

Palavras-chave: Cão; Neoplasia; Músculo estriado; Sistema urinário.

\begin{abstract}
Botryoid rhabdomyosarcoma is a variant of embryonic rhabdomyosarcoma and originates in striated muscle cells, or precursors of these cells, so they can start in parts of the body with the absence of striated muscle, such as the urogenital tract. Ser, the dogs affected are young up to two years old and the neoplastic masses look like a bunch of grapes. The objective of the present report was described as a case of botryoid rhabdomyosarcoma in a 16-month-old American Pittbull Terrier dog that presented a mass inside the urinary bladder, later diagnosed through histopathological and immunohistochemical examination. The mass was multilobulated, firm, pinkish to red with multifocal areas of necrosis and was adhered to the mucosa of the most cranial region of the urinary vesicle, emitting disseminated branches towards the bladder triangle. Microscopically, a neoplasm is discovered by two cell populations interspersed with a myxoid matrix, one composed of small, round cells, with scarce cytoplasm and hypercolored basophilic nucleus and the other by elongated cells, with eosinophilic and paracentral cytoplasm, both as having moderate and pleomorphism 2 mitosis
\end{abstract}


figures per field $(400 \mathrm{x})$. Increased cell density was observed below the bladder epithelium. A combination of complementary tests was conclusive for the diagnosis of neoplasia.

Keywords: Dog; Neoplasm; Striated muscle; Urinary system.

\section{Resumen}

El rabdomiosarcoma botrioide es una variante del rabdomiosarcoma embrionario y se origina en las células musculares estriadas, o precursoras de estas células, por lo que pueden comenzar en partes del cuerpo sin músculo estriado, como el tracto urogenital. Generalmente, los perros afectados son jóvenes de hasta dos años y las masas neoplásicas parecen un racimo de uvas. El objetivo del presente informe fue describir un caso de rabdomiosarcoma botrioide en un perro American Pittbull Terrier de 16 meses que presentaba una masa en el interior de la vesicula urinaria, posteriormente diagnosticada mediante examen histopatológico e inmunohistoquímico. La masa era multilobulada, firme, de color rosado a rojo con áreas multifocales de necrosis y estaba adherida a la mucosa de la region más craneal de la vejiga urinaria, emitiendo ramas diseminadas hacia el trígono vesical. Microscópicamente, la neoplasia está formada por dos poblaciónes celulares intercaladas con una matriz mixoide, una compuesta por células pequeñas y redondas, con escaso citoplasma eosinofílico y núcleo basófilo hipercoloreado y la otra por células alargadas, con citoplasma eosinofílico y núcleo paracentral, ambas poblaciónes celulares presentaban pleomorfismo moderado y 2 cifras de mitosis por campo (400 x). Se observó un aumento de la densidad celular debajo del epitelio de la vejiga. La combinación de exámenes complementarios fue concluyente para el diagnóstico de la neoplasia.

Palabras clave: Perro; Neoplasma; Músculo estriado; Sistema urinario.

\section{Introdução}

O rabdomiossarcoma é uma neoplasia infrequente em cães, originária de células musculares estriadas ou seus precursores. Existem quatro tipos histológicos de rabdomiossarcoma: alveolar, pleomórfico, embrionário e botrióide. Cada um desses tipos possui características próprias em relação a localização, idade e raça mais acometida (Castleman; Toplon; Clark, 2011; Caserto, 2013; Tochitani et al., 2013).

O rabdomiossarcoma embrionário é a forma mais comumente observada em animais, e o subtipo botrióide é frequentemente relatado em locais como o trato genitourinário e a cavidade oral de cães. Microscopicamente, ele é composto por células que remetem a embriogênese do músculo esquelético, e por isso podem estar presentes células variadas quanto a morfologia e grau de diferenciação. Estas células são envoltas em matriz mixóide e na camada mais próxima a mucosa, a celularidade tende a ser mais densa (Van Vechten; Goldschimidt; Wortman, 1990; Newton et al., 1995; Andrade, 2010; Meuten, 2017).

Há relatos de rabdomiossarcomas em diversas raças, tais como São Bernardos (Maxie; Newman, 2007), Golden Retriever (Bae et al., 2007) e Labrador (Costa et al., 2012). O presente estudo relata um caso de rabdomiossarcoma botrióide em um American Pittbull Terrier, raça sem relatos da neoplasia até o momento.

\section{Metodologia}

Um cão, American Pitbull Terrier, fêmea, de um ano e quatro meses de idade, deu entrada na Clínica veterinária da Universidade Federal do Vale do São Francisco (UNIVASF) com histórico de hematúria intensa e disúria há aproximadamente um mês.

Durante exame físico foi notado aumento de linfonodos submandibulares e cervicais superficiais e dor à palpação em região hipogástrica. $\mathrm{O}$ animal foi encaminhado ao serviço de ultrassonografia, que revelou espessamento da parede da vesícula urinária e presença de massa heterogênea de contornos irregulares na mucosa, apresentando vascularização interna. 
Figura 1. Imagem ultrassonográfica de rabdomiossarcoma em vesícula urinária de cão.

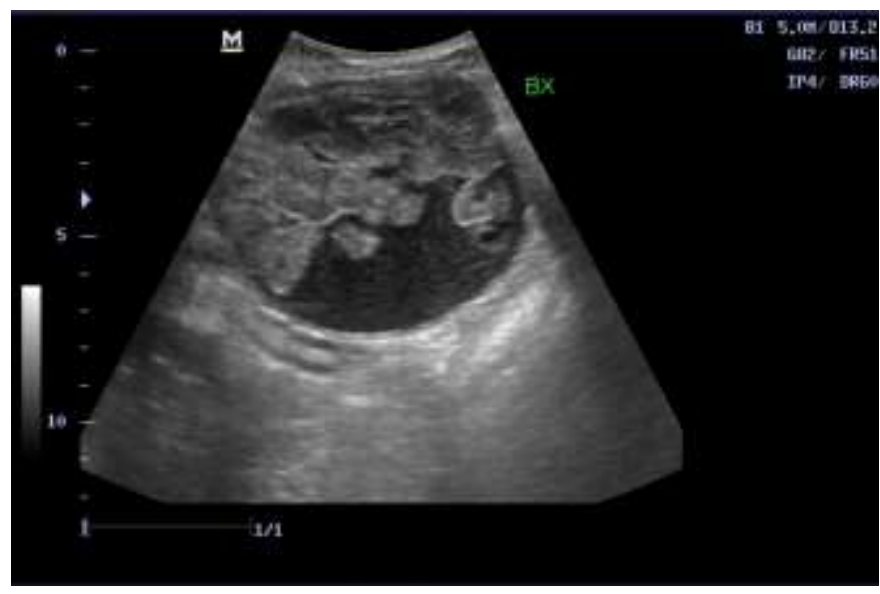

Clínica Veterinária da UNIVASF (2018).

O animal foi submetido a cirurgia para retirada da massa, porém devido à proporção que a tumoração já havia tomado, foi optado pela realização da biópsia incisional, pois não havia margens seguras para realização da exérese total do tumor, e o prognóstico era reservado. Dois dias depois da cirurgia, o tutor entrou em contato relatando que o animal estava em anúria e apatia e decidiu por realizar a eutanásia. O corpo do animal seguiu para autópsia.

Na análise do sistema urinário, notou-se congestão renal bilateral e uma discreta dilatação da pelve do rim direito. Não havia massa obstruindo ureteres e nem uretra. A vesícula urinária estava repleta de urina avermelhada e a massa localizada na mucosa do órgão media 12 × 9 × $4 \mathrm{~cm}$, estava aderida na região mais cranial da vesícula urinária e emitia ramificações disseminadas em direção ao trígono vesical, era firme, multilobulada, coloração que variava do róseo ao vermelho e múltiplas áreas de necrose com um tom esverdeado. Não foram encontrados focos de metástase em nenhum dos órgãos analisados.

Figura 2. Aspecto macroscópico de massa na vesícula urinária de cão American Pittbull Terrier, de 16 meses de idade. Nota-se uma massa multilobulada aderida na mucosa da vesícula urinária, com áreas centrais e periféricas de necrose.

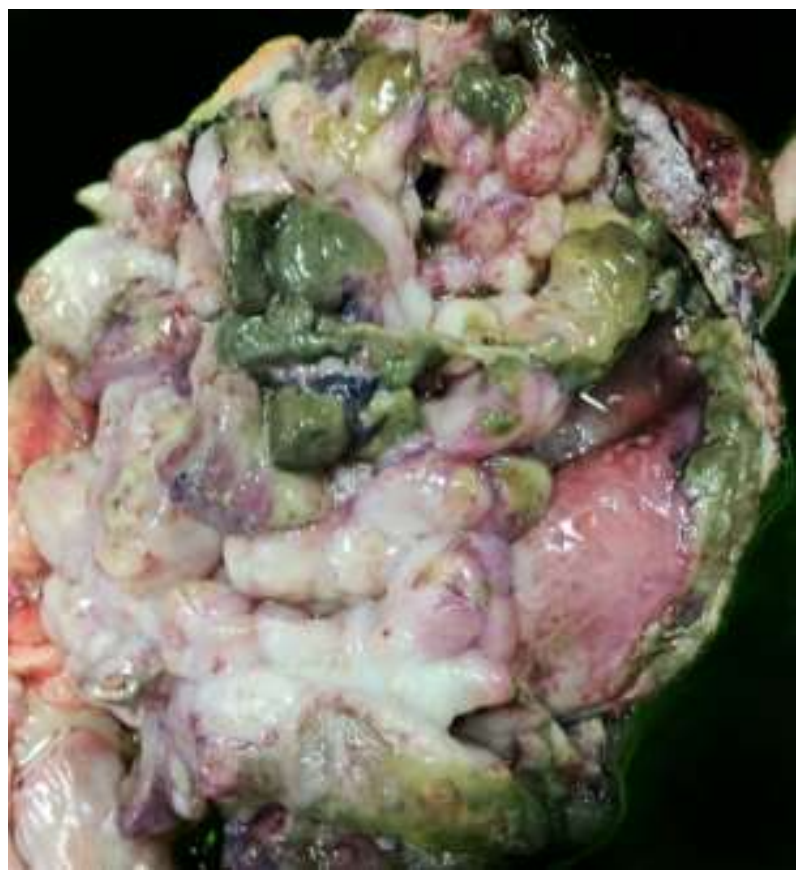

Fonte: Arquivo pessoal (2018). 
Amostras foram coletadas para realização de exame histopatológico, portanto foram mantidas em formaldeído $10 \%$ por 24 horas, submetidas a processamento histológico, embebidas em parafina e coradas em hematoxilina e eosina.

Microscopicamente, a neoplasia era constituída por duas populações celulares entremeadas a uma matriz mixóide, uma composta por células pequenas e redondas, com citoplasma escasso e núcleo basofílico com cromatina condensada e a outra por células alongadas, com citoplasma eosinofílico, núcleo paracentral, cromatina vesiculada, ambas as populações celulares possuiam anisocitose, anisocariose e pleomorfismo moderado e 2 figuras de mitose típicas por campo (400 x). Abaixo do epitélio havia múltiplos focos de aglomerados celulares. Áreas de necrose com infiltrado inflamatório misto (neutrófilos, macrófagos e linfócitos) e invasão e destruição do tecido não neoplásico adjacente foram observadas.

Figura 3. Fotomicroscopias de rabdomiossarcoma embrionário botrióide em cão, American Pittbull Terrier, de 16 meses de idade.
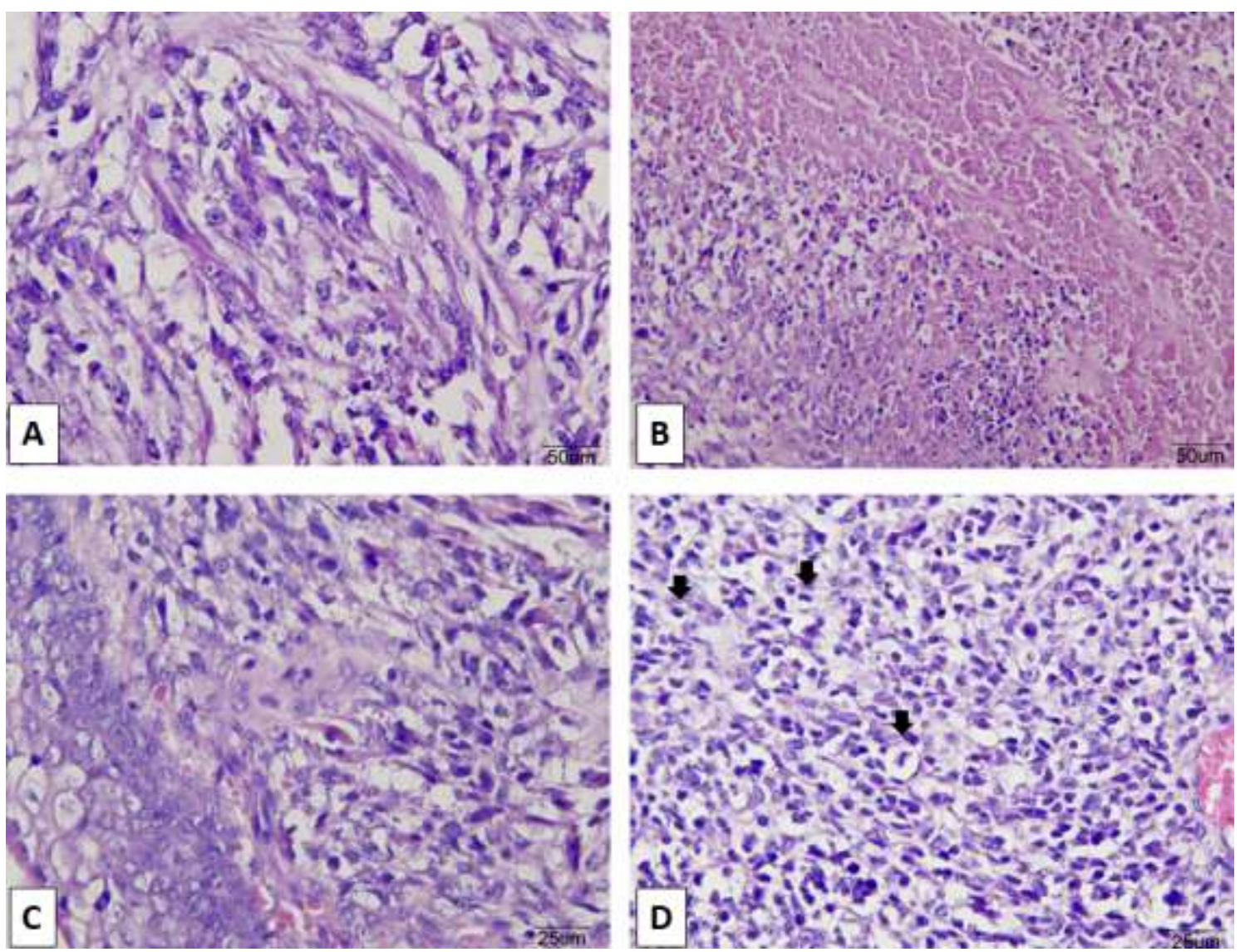

(A) Observa-se proliferação de dois tipos celulares compondo a neoplasia, células fusiformes e células arredondadas. (B) Há áreas de necrose, principalmente no centro da lesão, na figura observa-se aumento de eosinofilia tecidual com acentuada quantidade de células inflamatórias. (C) Sob o epitélio da mucosa vesical há uma camada densa de células neoplásicas, característica do rabdomiossarcoma botrióide. (D) Observase figuras de mitose típicas (setas).

Fonte: Arquivo pessoal (2018).

Exame imunoistoquímico foi realizado utilizando um painel com os seguintes anticorpos: actina do músculo liso alfa, desmina, mioglobina e MyoD. A marcação foi positiva quando utilizados os anticorpos desmina, mioglobina e MyoD, e negativa apenas com actina do músculo liso alfa. 
Tabela 1. Anticorpos utilizados na imuno-histoquímica para diagnóstico de rabdomiossarcoma.

\begin{tabular}{|c|c|}
\hline Anticorpos & Marcação \\
\hline Actina do músculo liso alfa & Negativo \\
\hline Desmina & Positivo \\
\hline Mioglobina & Positivo \\
MyoD & Positivo \\
\hline
\end{tabular}

Fonte: Autores.

Figura 4. Imuno-histoquímica de rabdomiossarcoma. Marcação em marrom, nas células neoplásicas, com os anticorpos Desmina (A), Mioglobina (B) e MyoD (C).

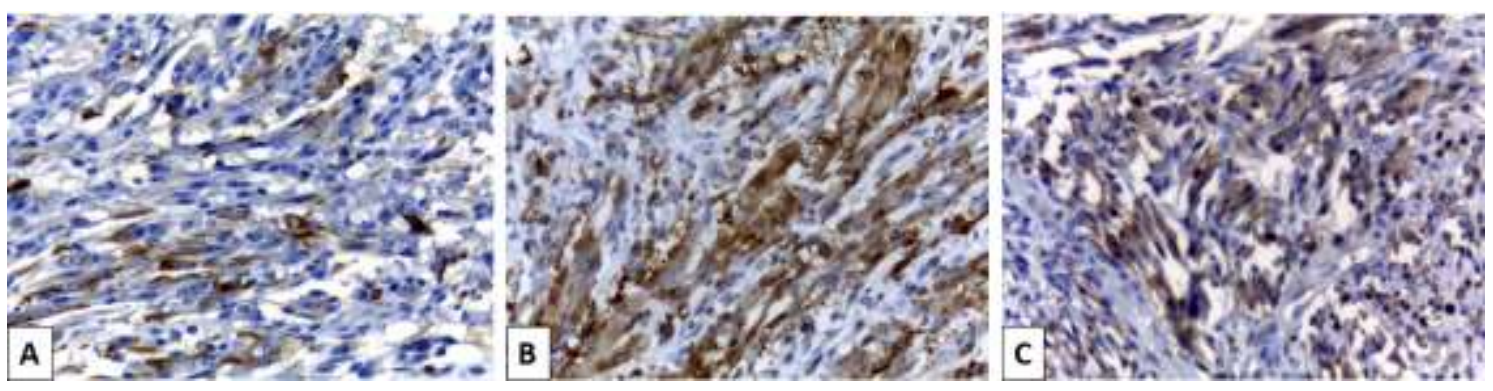

Fonte: Laboratório VetMol (2018).

\section{Discussão}

O rabdomiossarcoma embrionário botrióide já foi observado na vesícula urinária e descrito por diversos autores, pois apesar de ser uma neoplasia rara, quando ocorre, os locais de predileção são a vesícula urinária e a cavidade oral de cães (Kishimoto et al., 2017; Brockus; Myers, 2004; Kobayashi et al., 2004), principalmente cães jovens (menos de 2 anos) sugerindo uma origem embrionária da neoplasia (Caserto, 2013; Newman, 2016; Piazzolo; Da Cunha, 2020).

Dentre as raças mais acometidas estão os São Bernardos (Maxie; Newman, 2007), Golden Retrievers (KROTJE; Ware; Niyo, 1990; I.-H. Bae et al, 2007) e Labradores (Fecchio et al, 2004; Costa et al, 2012). No presente relato, o cão era da raça American Pitbull Terrier, na qual não foram encontrados outros relatos de rabdomiossarcoma. Porém existe a semelhança de todas serem raças de cães de grande porte (Newman, 2016).

$\mathrm{Na}$ análise microscópica observamos duas populações celulares morfologicamente diferentes fazendo parte da mesma neoplasia. A literatura afirma que essa característica é observada em rabdomiossarcomas, justificando que as populações celulares observadas na neoplasia apresentam diferentes fases de desenvolvimento do músculo estriado, portanto a morfologia celular pode variar desde mioblastos redondos e imaturos a células tubulares multinucleadas que se assemelham as células musculares em desenvolvimento (Caserto, 2013, Devriendt et al, 2016). Outra característica histológica observada neste trabalho e citada pela literatura é a proliferação celular mais densa sob o epitélio da mucosa e menor densidade celular com maior quantidade de matriz mixóide observada à medida que as células se afastam da mucosa (Van Vechten; Goldschimidt; Wortman, 1990).

$\mathrm{Na}$ variante botrióide do rabdomiossarcoma raramente ocorrem metástases, diferente das outras variantes onde o índice metastático pode chegar a 50\%. Dessa forma, como esperado, não foram encontrados focos metastáticos em nenhum dos órgãos do animal relatado. (Caserto, 2013; Devriendt et al, 2016).

O painel de anticorpos utilizados no exame imuistoquímico confirmou a origem muscular das células. $\mathrm{O}$ anticorpo anti actina do músculo liso alfa tem marcação positiva no músculo liso, miofibroblastos, células miointimais e células mesoteliais 
reativas, células que não foram identificadas nesse caso. Dessa forma, a identificação do antígeno em sarcomas não pode confirmar o diagnóstico de rabdomiossarcoma, mas é utilizado porque se houver marcação negativa, pode-se excluir leiomiossarcomas e neoplasias malignas contendo miofibroblastos, direcionando o diagnóstico para rabdomiossarcoma (Caserto, 2013).

Desmina é uma proteína de filamento intermediário presente em todas as células musculares e em todas as fases de diferenciação celular (Radi, 2006), portanto é bastante utilizado como marcador muscular específico (Fletcher; Jorgensen, 2000), sendo útil na identificação de sarcomas indiferenciados. O resultado encontrado na neoplasia desse caso foi desmina positivo, assim como relatado por Takiguchi et al, 2002 e Gileem et al, 2018, em outros casos de rabdomiossarcoma.

Uma variedade de estudos mostra que rabdomiossarcomas foram confirmados usando anticorpo anti-mioglobina, o que demonstra a eficácia e utilidade do anticorpo, apesar de algumas desvantagens (Sarnelli; Grassi; Romagnoli, 1994; Kim et al., 1996; Bae et al., 2007). Uma dessas desvantagens é que o músculo esquelético imaturo, bem como as células tumorais pouco diferenciadas geralmente têm expressão rara de mioglobina, pois esta é considerada um marcador muscular de maturação tardia. Então ao interpretar o resultado da imunohistoquímica, deve-se levar em consideração que rabdomiossarcomas indiferenciados podem ser negativos para mioglobina (Caserto, 2013). No presente estudo, o anticorpo foi utilizado e se mostrou positivo nas células neoplásicas, direcionando o diagnóstico, pois tal anticorpo tem reação positiva, principalmente em células musculares cardíacas e esqueléticas (Caserto, 2013).

Expressão de MyoD em rabdomiossarcomas já foi relatada por diversos autores, e atualmente, é um anticorpo bastante utilizado para fins diagnóstico deste tipo de neoplasia muscular (Kobayashi et al., 2004, Tuohy et al., 2021). Está entre os marcadores mais recentes para o diagnóstico de rabdomiossarcoma, e é sensível e específico para tal neoplasia. MyoD é um fator de transcrição detectado em células musculares esqueléticas e está envolvido na transformação mioblástica de células-tronco mesenquimais, e por isso não é detectado nas células musculares maduras (Folpe, 2002, Meuten, 2017).

\section{Conclusão}

A característica aparência macroscópica e a localização da neoplasia, bem como os achados histopatológicos, e as marcações imunohistoquímicas, nos permitiram o diagnóstico de rabdomiossarcoma embrionário botrióide de vesícula urinária em um cão da raça American Pittbull Terrier.

Faz- se necessário incluir o rabdomiossarcoma embrionário botrióide no diagnóstico diferencial de neoformação em vesícula urinária de cães jovens, incluído a raça Pitttbul Terrier, na qual a neoplasia referida ainda não foi relatada, contudo apresentou o mesmo comportamento, evolução, e aspectos diagnósticos descritos em literatura.

\section{Referências}

Andrade C. R., Takahama Junior A., Nishimoto I. N., Kowalski L. P., \& Lopes M. A. (2010). Rhabdomyosarcoma of the head and neck: a clinicopathological and immunohistochemical analysis of 29 cases. Brazilian Dental Journal, 21(1), 68-73. https://doi.org/10.1590/S0103-64402010000100011.

Bae I.-H., Kim Y., Pakhrin B., You M. H., Hwang C. Y., Kim J. H., \& Kim D. Y. (2007). Genitourinary Rhabdomyosarcoma with Systemic Metastasis in a Young Dog. Veterinary Pathology, 44(4), 518-520. 10.1354/vp.44-4-518.

Breshears M. A. \& Confer A. W. (2017). Pathologic Basis of Veterinary Disease. (6a ed.), Mosby/Elsevier.

Brockus, C. W. \& Myers, R. K. (2004). Multifocal rhabdomyosarcomas within the tongue and oral cavity of a dog. Veterinary Pathology, 41(3), 273-274. $10.1354 / \mathrm{vp} .41-3-273$

Caserto B. G. (2013). A Comparative Review of Canine and Human Rhabdomyosarcoma With Emphasis on Classification and Pathogenesis. Veterinary Pathology, 50(5), 806-826. 10.1177/0300985813476069.

Castleman, W. L., Toplon, D. E., \& Clark, C. K. (2011). Rhabdomyosarcoma in 8 horses. Veterinary Pathology, 48, 1144-50. 10.1177/0300985810395785. 
Charadán, A. M. S., Baglans D. S., Hechavarría J. P., Agüero H. B., Preval M. M., Brooks O. I., \& Frómeta R. R. (2005). Sarcoma botrioideo de vagina. Informe de un caso. Revista Información Científica, 46(2). https://www.redalyc.org/articulo.oa?id=551757340009

Cooper B. J. \& Valentine B. A. (2017). Tumors in Domestic Animals. (5th ed.), John Wiley \& Sons.

Costa, T. C., Torres A. A. A., Ibañez J. F., Olenscki T. J., Nobre C. S., Reis Filho N. P., \& Calderón C. (2012). Papel do exame citopatológico como método diagnóstico em um caso de rabdomiossarcoma embrionário em cão. Revista Científica Eletrônica De Medicina Veterinária, http://faef.revista.inf.br/imagens_arquivos/arquivos_destaque/66XQmdaXrKo8cmW_2013-6-25-16-55-4.pdf.

Devriendt N., Van Brantegem L., Willems A., Raes E., \& Rooster H. (2016). Embryonal Rhabdomyosarcoma of the Oesophagus in a Young Dog. Journal of Comparative Pathology. 156(1), 21 - 24. 10.1016/j.jcpa.2016.10.002.

Fecchio, R. S., Petri B. S. S., Coutinho A., Kleeb S. R., Xavier J. G., \& Zanco N. A. (2004). Uso da imunohistoquímica no diagnóstico de rabdomiossarcoma em cavidade oral de cão: relato de caso. III ONCOVET - Simpósio de Oncologia Veteriária. São Paulo/SP. http://www.usp.br/locfmvz/antigo/download/Artigos/Rabdomiossarcoma.pdf.

Fletcher M. \& Jorgensen D. (2000). Leiomyosarcomas of the oral cavity: an unusual topographic subset easily mistaken for nonmesenchymal tumours. Histopathology, 36(30, 210 - 220. 10.1046/j.1365-2559.2000.00814.x.

Folpe A. L. (2002). MyoD1 and Miogenin Expression in Human Neoplasia: A Review and Update. Advances in Anatomic Pathology, 9(3), $198-203$. 10.1097/00125480-200205000-00003.

Gillem J. M., Sullivan L., \& Sorenmo K. U. (2018). Diagnosis and Multimodal Treatment of Metastatic Maxillofacial Juvenile Embryonal Rhabdomyosarcoma in a Young Golden Retriever. American Animal Hospital Association, p. 54-55. 10.5326/JAAHA-MS-6662.

Kim D. Y., Hodgin E. C., Cho D. Y., Varnado J. E. (1996). Juvenile Rhabdomyosarcomas in Two Dogs. Veterinary Pathology, 33(4). $10.1177 / 030098589603300416$.

Kishimoto, T.E., Yashima S., Nakahira R., Onozawa E., Azakami D., Ujike M., Ochiai K., Ishiwata T., Takahashi K., \& Michishita M. (2017). Identification of tumor initiating cells derived from two canine rhabdomyosarcoma cell lines. The Journal of Veterinary Medical Science, 79,1155-1162. 10.1292/jvms.16-0412.

Kobayashi M., Sakai H., Hirata A., Yonemaru K., Yanai T., Watanabe K., Yamazoe K., Kudo T., \& Masegi T. (2004). Expression of myogenic regulating factors, Myogenin and MyoD, in two canine botryoid rhabdomyosarcomas. Veterinary Pathology, 41(3), 275-277. 10.1354/vp.41-3-275.

Krotje L. J., Ware W. A., \& Niyo Y. (1990). Intracardiac rhabdomyosarcoma in a dog. Journal of the American Veterinary Medical Association, 197(3), 368371. Retrieved from: https://pubmed.ncbi.nlm.nih.gov/2391275/

Maxie, M. G. \& Newman, S. J. (2007). Pathology of Domestic Animals. (5a ed.), Saunders Elsevier.

Meuten, D. J. (2017). Tumors in domestic animals. (5a ed.), John Wiley \& Sons.

Newton, W. A., Gehan E. A., Webber B. L., Marsden H. B., Van Unnik A. J., Hamoudi A. B., Tsokos M. G., Shimada H., Harms D., \& Schimidt D. (1995). Classification of Rhabdomyosarcomas and related sarcomas. Cancer, Philadelphia, 76(6), 1073-1085. 10.1002/1097-0142(19950915)76:6<1073::aidcncr2820760624>3.0.co;2-1.

Newman S. J. (2016). Bases da patologia em veterinária. (5a ed.), Elsevier.

Piazzolo M., \& Da cunha O. (2020). Rabdomiossarcoma embrionário em vesícula urinária de cão com oito meses de idade - relato de caso. A pesquisa nos diferentes campos da medicina veterinária. Editora Atena, cap. 17, p. 132-137. https://www.finersistemas.com/atenaeditora/index.php/ad min/api/artigoPDF/42816.

Radi Z. A. (2006). Auricular Rhabdomyosarcoma in a Rat. Journal of Veterinary Medicine, 53(5), 246-248. 10.1111/j.1439-0442.2006.00829.x.

Sarnelli R., Grassi F., \& Romagnoli S. (1994). Alveolar Rhabdomyosarcoma of the Greater Omentum in a Dog. Veterinary Pathology, 31, 473-475. https://doi.org/10.1177/030098589403100412.

Takiguchi M., Watanabe T., Okada H., Kudo H., Yamada K., Yasuda J., \& Hashimoto A. (2002). Rhabdomyosarcoma (botryoid sarcoma) of the urinary bladder in a Maltese. Journal of Small Animal Practice, 43(6), 269-271. 10.1111/j.1748-5827.2002.tb00072.x.

Tochitani T., Matsumoto I., Hoshino K., Toyosawa K., Kouchi M., Koujitani T., Kimura J., \& Funabashi H. (2013). Spontaneous rhabdomyosarcoma in a common marmoset (Callithrix jacchus). Journal of Toxicologic Pathology, 26,187-191. 10.1293/tox.26.187

Tuohy J. L., Byer B. J., Royer S., Keller C., Nagai-Singer M. A., Regan D. P., \& Seguin B. (2021). Evaluation of Myogenin and MyoD1 as Immunohistochemical Markers of Canine Rhabdomyosarcoma. Veterinary Pathology, 58, 516 - 526. 10.1177/0300985820988146.

Van Vechten, M., Goldschmidt, M. H., \& Wortman, J. A. (1990). Embryonal rhabdomyosarcoma of the urinary bladder in dogs. Compendium on Continuing Education for the Practicing Veterinarian, 12(6), 783-786. https://inis.iaea.org/search/searchsinglerecord.aspx?recordsFor=SingleRecord\&RN=45092630. 